\title{
Waist Circumference Measured by Bioelectrical Impedance Analysis Is Interchangeable with Manual Measurement: Increased Waist Circumference Is Associated with Locomotive Syndrome Risk
}

\author{
Satoshi Tanaka, ${ }^{1}$ Kei Ando, ${ }^{1}$ Kazuyoshi Kobayashi $\left(\mathbb{D},{ }^{1}\right.$ Taisuke Seki, ${ }^{1}$ Shinya Ishizuka, ${ }^{1}$ \\ Masaaki Machino, ${ }^{1}$ Masayoshi Morozumi, ${ }^{1}$ Shunsuke Kanbara, ${ }^{1}$ Sadayuki Ito, ${ }^{1}$ \\ Taro Inoue, ${ }^{1}$ Naoki Ishiguro, ${ }^{1}$ Yukiharu Hasegawa, ${ }^{2}$ and Shiro Imagama $\mathbb{D}^{1}$ \\ ${ }^{1}$ Department of Orthopaedic Surgery, Nagoya University Graduate School of Medicine, Nagoya, Aichi, Japan \\ ${ }^{2}$ Department of Rehabilitation, Kansai University of Welfare Science, Osaka, Japan
}

Correspondence should be addressed to Shiro Imagama; imagama@med.nagoya-u.ac.jp

Received 30 May 2019; Revised 3 August 2019; Accepted 4 September 2019; Published 25 September 2019

Academic Editor: Zbigniew Gugala

Copyright ( ) 2019 Satoshi Tanaka et al. This is an open access article distributed under the Creative Commons Attribution License, which permits unrestricted use, distribution, and reproduction in any medium, provided the original work is properly cited.

\begin{abstract}
Objectives. The importance of preventive medicine in an aging society is increasing. Locomotive syndrome (LS) is attracting increasing attention. Recently, advances in bioelectrical impedance analysis (BIA) devices have made it possible to automatically measure waist circumference (WC). Nevertheless, there have been no reports evaluating the agreement or interchangeability between WC measured manually and using BIA. Therefore, we aimed to perform these analyses in the context of health checkups and investigated the associations with LS risk. Methods. We enrolled 597 participants who underwent the following: two-step tests and stand-up tests; evaluations using a 25-question geriatric locomotive function scale for the LS risk test; anthropometric marker measurements including WC measured by manual and BIA; and measurements of total cholesterol and triglycerides. We used Bland-Altman analysis to calculate agreement and interchangeability of the WC measurement using BIA and the manual method. A statistical comparative study was then conducted between normal and LS risk groups. Subsequently, significant risk factors for LS were investigated using multivariate analysis. Results. The Bland-Altman analysis showed that bias (BIA-manual) was negative overall $(-2.024)$, for males $(-1.418)$ and for females $(-2.460)$, suggesting underestimation using BIA compared with manual measurements. Interchangeability was found between WC measurement by BIA and by manual methods, because the percentage error was less than $15 \%$ overall $(12.3 \%)$, for males $(10.2 \%)$ and for females $(13.8 \%)$. Univariate analysis showed that WC was significantly higher in the LS risk group than in the normal group. Multivariate analysis adjusted for confounding factors showed that increase in WC significantly correlated with LS risk. Conclusions. BIA and manual methods for measuring WC are interchangeable. The increase in WC measured by BIA was significantly associated with LS risk. It is important to continue focusing on increased WC and early detection of LS risk.
\end{abstract}

\section{Introduction}

In Japan, the average life expectancy has increased in parallel with advanced medical advances. The importance of preventive medicine is implied by phenomena such as prolonged hospitalization due to decreased mobility in elderly people.
In 2007, the Japanese Orthopaedic Association (JOA) proposed the concept of "locomotive syndrome" (LS). LS is a condition in high-risk individuals with musculoskeletal disease who are likely to require nursing care at some point [1]. In 2013, the JOA proposed the following tests to estimate the risk of LS: the two-step test; the stand-up test; and a 25question geriatric locomotive function scale (GLFS-25). On 
the basis of these test results, mobility and stage of LS can be determined. LS risk levels are categorized into two stages: 1 and 2. Risk level 1 represents the population in whom movement function has started to decline and for whom measures to prevent deterioration to LS should be instituted $[2,3]$. Early diagnosis of LS risk level is important to permit treatment of these conditions, and investigation of the relationships with LS risk from a broad perspective is necessary $[4,5]$.

Previously, we reported that waist circumference (WC) was significantly associated with LS in elderly females, and this association was more significant than that for other obesity-related parameters [6]. However, in that report, LS risk was not evaluated; moreover, males were not included.

Body composition analysis using bioelectrical impedance analysis (BIA) easily measures water content and body fat mass; it is commonly performed in the context of general medical examinations [7-9]. In recent years, the evolution of BIA devices has been remarkable such that devices can automatically measure not only WC but also the circumference of the neck, chest, and hip in a short period of time. Nevertheless, there have been no reports validating WC by BIA and manual methods, particularly in terms of interrater reliability, agreement, and interchangeability.

Therefore, the purpose of this study was to validate WC measurement by BIA and by manual methods. For this purpose, Spearman correlation and interclass correlation coefficient (ICC) as well as Bland-Altman analysis [10] was used to examine whether these two measurements are interchangeable. Then, we examined whether WC measurement by BIA correlates with LS risk according to sex in a large-scale prospective general health checkup population.

\section{Materials and Methods}

2.1. Participants. The study participants were volunteers who underwent health checkups supported at the local government of the town of Yakumo, Japan, in 2016-2017. Yakumo town has a population of approximately 17,000 people, of which $28 \%$ are over 65 years old. More people are engaged in agriculture and fishery than those in urban areas. Checkups have been conducted annually in this town since 1982. The checkup consists of voluntary orthopedic and physical function examinations, internal medical examinations, and psychological tests, as well as a health-related quality of life (QoL) survey [11-14]. In this study, participants underwent LS risk tests, anthropometric marker measurements including WC measured manually and by BIA, and blood test. The BIA measurement conditions such as consumption of food and beverage were based on previous report [15] as much as possible, so they underwent these evaluations on an empty stomach. These tests and measurements were performed in the order of blood test, BIA, and LS risk tests. Exclusion criteria were as follows: history of spine and limb joint surgery; severe knee injury; severe osteoarthritis; history of fracture in the hip and spine; neurological disorders; severe mental illness; diabetes; kidney or heart disease; not empty stomach; and severe disability in walking or standing or any dysfunction of the central or peripheral nervous systems.

Among the 1094 individuals who underwent the health checkups, 597 participants (250 males and 347 females) met the selection criteria.

The study protocol was approved by the ethics committee of human research and the institutional review board of our university (No. 2014-0207). All participants provided written informed consent prior to participation. The study procedures were carried out in accordance with the principles of the Declaration of Helsinki.

2.2. GLFS-25. The GLFS-25 is a self-administered questionnaire consisting of 25 items graded on a 5-point scale, from no impairment ( 0 points) to severe impairment (4 points) $[16,17]$. The sum of the 25 -item scores yields a total possible score between 0 and 100, with increasing values indicating increasing severity of LS. The validity and reliability of this instrument have been reported to be satisfactory, with LS defined by a score $\geq 16$ points and non-LS as $\leq 15$ points. For this study, we used "Locomo 25," the Japanese version of the GLFS-25.

2.3. Two-Step Test. The two-step test measures stride length to evaluate walking ability, including muscle strength, balance, and flexibility of the lower limbs [3]. Subjects stood with the toes of both feet behind a starting line and were then instructed to take two long steps (as long as possible) and then align both feet. The length of the two steps from the starting line to the tips of the toes at the point where the subject stopped was measured. The two-step test score was calculated as the length of the two steps $(\mathrm{cm})$ divided by height $(\mathrm{cm})$.

2.4. Stand-Up Test. In the stand-up test, leg strength was assessed by having the subject stand up on one or both legs from a specified height. The subject stood up from each of four seats of heights $(40,30,20$, and $10 \mathrm{~cm})$ in descending height order, first with both legs then with one leg. A subject who could stand up without leaning back to gain momentum and could maintain the posture for $3 \mathrm{~s}$ was considered to have passed that height level [3]. In this study, a subject who was unable to stand up on one leg (right or left) from a height of $40 \mathrm{~cm}$ was considered to have failed the test.

2.5. LS Risk Test. The JOA defines two stages of LS risk. LS risk stage 1 is defined as a two-step test score $<1.3$, difficulty with one-leg standing from a $40 \mathrm{~cm}$ seat in the stand-up test (either leg), or a GLFS-25 score $\geq 7$. Subjects meeting any of these criteria were diagnosed as starting to experience a decline in mobility. LS risk stage 2 is defined as a two-step test score $<1.1$, difficulty with standing from a $20 \mathrm{~cm}$ seat using both legs in the stand-up test, or a GLFS- 25 score $\geq 16$. Subjects meeting any of these criteria were diagnosed as having progression of decline in mobility. In this study, subjects who met the criteria for LS risk test stage 1 or 2 were 
defined as LS risk subjects and the other subjects were defined as normal $[4,5]$.

2.6. Anthropometric Measurements. Anthropometric data including weight, body mass index (BMI), percent body fat (PBF), WC, and appendicular skeletal muscle mass (aSMI) as muscle mass were measured using BIA. The Inbody 770 BIA unit (Inbody Co., Ltd., Seoul, Korea), used to differentiate tissues (such as fat, muscle, and bone) based on their electrical impedance, was utilized [7-9, 18-20]. The accuracy of this device has been reported by various approaches $[21,22]$. Individuals grasped the handles of the analyzer, in which electrodes are embedded, and stood on the platform, with the sole of the feet in contact with the electrodes (two electrodes for each foot and hand). BMI was calculated using the following formula: weight $(\mathrm{kg}) /$ height $^{2}\left(\mathrm{~m}^{2}\right)$. WC by BIA was calculated automatically using the Inbody 770 BIA device. The aSMI was calculated using the following formula: aSMI $=$ arm and leg skeletal muscle mass $(\mathrm{kg}) /$ height $^{2}\left(\mathrm{~m}^{2}\right)$ [23]. WC was measured manually at the level of the umbilicus at the end of gentle expiration with the subject standing using a nonstretchable measuring tape and recorded in centimeters to the nearest millimeter [6].

2.7. Blood Test. We obtained venous blood samples to measure total cholesterol and triglycerides (related to metabolic syndrome). Biochemical analyses of the blood samples were performed using an autoanalyzer (JCA-RX20; Nihon Denshi, Tokyo, Japan).

2.8. Statistical Analysis. Continuous variables were expressed as means and standard deviations (SDs), while categorical variables were expressed as percentages. The Kolmogorov-Smirnov test was used to evaluate the normality of the distribution of the data, so the Mann-Whitney $U$ test and Fisher's exact test were used to evaluate betweengroup differences, as appropriate for the data distribution. The correlations between manual methods and BIA for measuring WC were examined using the Spearman $r$ and ICC (absolute agreement, two-way random, and single measures). The following cutoff values were used to interpret Spearman correlations: $r<0.20=$ very weak; 0.20 to $0.39=$ weak; 0.40 to $0.59=$ moderate; 0.60 to $0.79=$ strong; and 0.80 to $1.0=$ very strong [23]. The cutoff values to interpret the ICC were as follows: $<0.20=$ slight; 0.20 to 0.39 = fair; 0.40 to $0.59=$ moderate; 0.60 to $0.79=$ substantial; and 0.80 to $1.0=$ almost perfect [24]. To examine the level of agreement between the manual and BIA measurements, Bland-Altman analysis was used [10]. The mean of the difference between measurements (BIA-manual) was defined as bias, and standard deviation (SD) was also used to calculate 95\% confidence limits of agreement (LOA, bias $\pm 1.96 \mathrm{SD}$ ). Bland-Altman plots graphically display the mean of the two measured values (WC measurement using BIA and the manual method) on the $X$-axis and the difference (BIA-manual) between measured values on the $Y$ axis. Whether BIA is interchangeable with the manual method was defined using percentage error (the ratio of 1.96 SD to the mean value of the manual) as $15 \%$ or less [25]. To determine the factors associated with LS risk among the variables that exhibited differences $(P<0.25)$ in the univariate analyses, logistic regression analysis using a stepwise method was performed using the aforementioned variables as covariates. A $P$ value of $<0.05$ was considered significant in all analyses. Statistical analyses were conducted using JMP Pro version 13.1 for Mac (SAS Institute Inc., Cary, NC, USA).

\section{Results}

The average age of the 579 participants was 64.6 years (range, 40-88; SD: 10.1 years), the average BMI was $23.5 \mathrm{~kg} / \mathrm{m}^{2}$, and the average PBF was $29.1 \%$.

Table 1 shows the demographic, anthropometric, blood test, and LS risk prevalence data. There were significant differences between males and females for all variables.

Table 2 shows Spearman $r$, ICC, and Bland-Altman analysis results for the values of $\mathrm{WC}$ measured by two methods: manual and BIA. The Spearman $r$ was also illustrated in Figure 1, and the Bland-Altman plots are shown in Figure 2. Overall, and in males and females, WC measured by BIA showed a very strong correlation with WC measured manually based on the Spearman $r$ and almost perfect agreement based on ICC. The Bland-Altman analysis showed that bias (BIA-manual) was negative overall $(-2.024)$, for males $(-1.418)$ and for females (-2.460), suggesting an underestimation of the BIA compared with the manual methods. We also found that the difference was larger in females than in males. We found that the agreement between the WC measurements using BIA and manual methods were more consistent in males than in females. Interchangeability was found between the WC measured by BIA and the manual method because the percentage error was less than $15 \%$ overall $(12.3 \%)$, for males $(10.2 \%)$ and for females $(13.8 \%)$.

Table 3 shows the comparisons between the normal group and the LS risk group according to sex. For overall participants, a significant difference was observed in terms of age $(P<0.001)$, sex $(P=0.010), \operatorname{BMI}(P<0.001)$, PBF $(P<0.001)$, WC by BIA $(P=0.003)$, and triglycerides $(P=0.019)$ between the groups. For males, there were significant difference in terms of age $(P=0.002)$, BMI $(P=0.041)$, PBF $(P=0.003)$, and WC by BIA $(P=0.027)$; for females, there were significant differences in terms of age $(P=0.007)$, weight $(P<0.001)$, BMI $(P<0.001)$, PBF () , aSMI $(P=0.006)$, WC by BIA $(P<0.001)$, and triglycerides $(P=0.006)$.

Table 4 shows the result of the logistic regression model for LS risk in all participants. WC by BIA $(P<0.001)$, age $(P<0.001)$, female $(P<0.001)$, and total cholesterol $(P=0.016)$ were significantly associated with LS risk.

Table 5 shows the results of the logistic regression model according to sex. Only WC by BIA and age were significantly associated with LS risk in both males and females. From these results, it was shown that the increase in WC measurement by BIA significantly correlated LS risk as well as increase in age. 
TABLE 1: Demographic, anthropometric, blood test, and LS risk prevalence data of the study participants.

\begin{tabular}{|c|c|c|c|c|}
\hline Variables & Total $(N=597)$ & Male $(N=250)$ & Female $(N=347)$ & $P$ value \\
\hline Age (years) & $64.6(10.1)$ & $66.5(9.3)$ & $63.3(10.4)$ & $<0.001$ \\
\hline Height $(\mathrm{cm})$ & $158.1(8.5)$ & $165.0(6.1)$ & $153.1(6.2)$ & $<0.001$ \\
\hline Weight (kg) & $59.2(11.6)$ & $66.9(10.7)$ & $53.7(8.8)$ & $<0.001$ \\
\hline $\operatorname{BMI}\left(\mathrm{kg} / \mathrm{m}^{2}\right)$ & $23.6(3.5)$ & $24.5(3.3)$ & $22.9(3.5)$ & $<0.001$ \\
\hline PBF (\%) & $29.1(6.9)$ & $25.2(5.7)$ & $31.9(6.3)$ & $<0.001$ \\
\hline $\operatorname{aSMI}\left(\mathrm{kg} / \mathrm{m}^{2}\right)$ & $6.7(1.0)$ & $7.6(0.8)$ & $6.1(0.7)$ & $<0.001$ \\
\hline WC by manual $(\mathrm{cm})$ & $81.8(10.1)$ & $86.3(8.9)$ & $78.5(9.6)$ & $<0.001$ \\
\hline WC by BIA $(\mathrm{cm})$ & $79.8(9.6)$ & $84.9(9.5)$ & $76.1(7.9)$ & $<0.001$ \\
\hline Total cholesterol (mg/dL) & $208.6(32.7)$ & $203.6(33.6)$ & $212.1(31.6)$ & 0.009 \\
\hline Triglycerides (mg/dL) & $107.3(65.4)$ & $122.4(82.4)$ & $96.5(46.9)$ & $<0.001$ \\
\hline Prevalence of LS risk (\%) & $54.6 \%$ & $48.4 \%$ & $59.1 \%$ & 0.010 \\
\hline
\end{tabular}

Evaluated using the Mann-Whitney $U$ test, Fisher's exact test. Parameter values are shown as means (standard deviations) or numbers. Bold values indicate a significant difference. PBF and aSMI were measured using Inbody 770 BIA unit. BMI, body mass index; PBF, percent body fat; WC, waist circumference; BIA, bioelectrical impedance analysis; aSMI, appendicular skeletal muscle index; LS, locomotive syndrome.

TABLE 2: Correlation coefficient ( $r$, ICC, and Bland-Altman analysis in WC measured by two methods: manual and BIA.

\begin{tabular}{lccc}
\hline & Total & Male & Female \\
\hline Spearman $r$ & $0.871^{* * *}$ & $0.875^{* * *}$ & $0.830^{* * *}$ \\
ICC & 0.930 & 0.940 & 0.896 \\
Bland-Altman analysis & & & -1.418 \\
Bias (BIA-manual) & -2.024 & 4.395 & -2.460 \\
SD & 5.025 & -10.03 to 7.20 & 5.398 \\
$95 \%$ LOA & -11.87 to 7.83 & 10.2 & -13.04 to 8.12 \\
Percentage error (\%) & 12.3 & 13.8 \\
\hline
\end{tabular}

${ }^{* * *} P<0.001 . r$, correlation coefficient; ICC, interclass correlation coefficients; WC, waist circumference; BIA, bioelectrical impedance analysis; SD, standard deviation; LOA, limits of agreement.

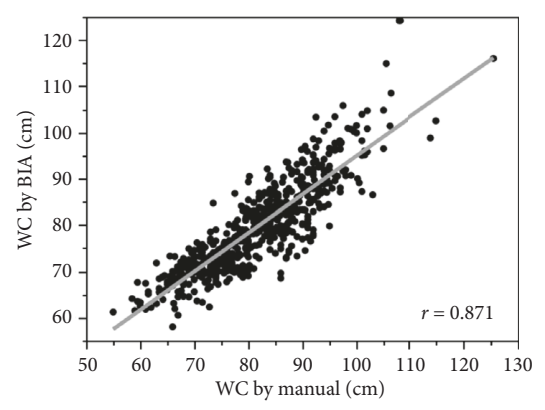

(a)

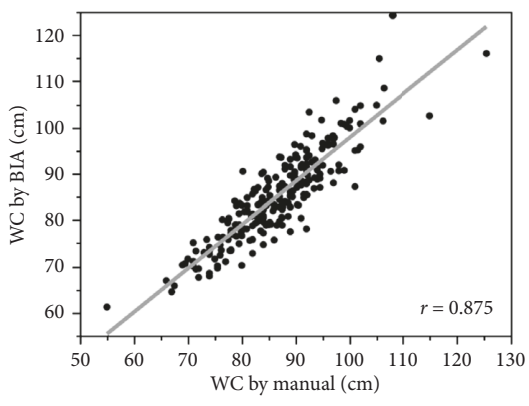

(b)

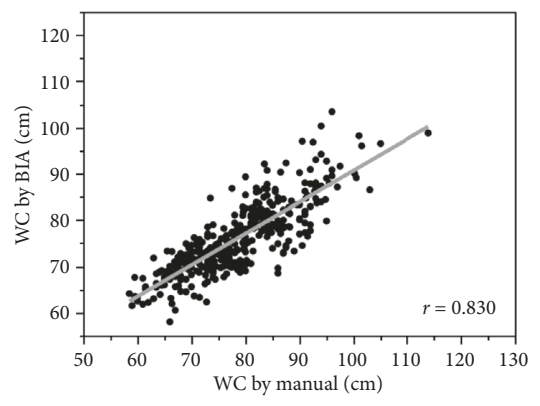

(c)

FIGURE 1: Scatter plot of WC by manual method and BIA. WC measured by BIA had significantly very strong positive correlation with that of the manual method. (a) Total $(r=871, P<0.001)$. (b) Male $(r=0.875, P<0.001)$. (c) Female $(r=0.830, P<0.001)$.

\section{Discussion}

WC is an obesity-related parameter that has been reported to be associated with various diseases and conditions [26-28]. Currently, WC measurement is performed manually. In large-scale health checkups, manual measurements of many participants within a limited time may cause interrater error because of increases in the number of measurers. To solve such problems, there is a need for a device that has a high degree of agreement with manual measurement and can be used to perform automatic measurements in a short period time. In recent years, BIA, a portable device with no exposure, has been used for measurement of body composition and anthropometric markers. We also use it in the context of health checkups. Because BIA can obtain a large amount of data in a short period of time using one measurement, it has the great advantages of reducing labor, time, and interrater errors compared to those associated with manual measurement. Advances in technology have also generated BIA devices that can automatically measure trunk circumferences including WC. Nevertheless, there has been no report on the validation of BIA with respect to manual measurement values. The present study was the first to validate WC measured by these two measurement methods 


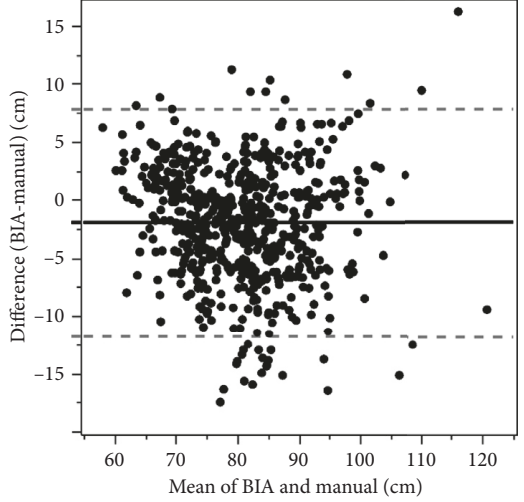

(a)

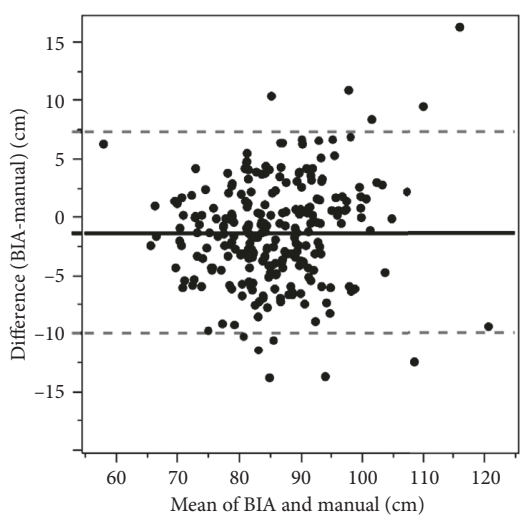

(b)

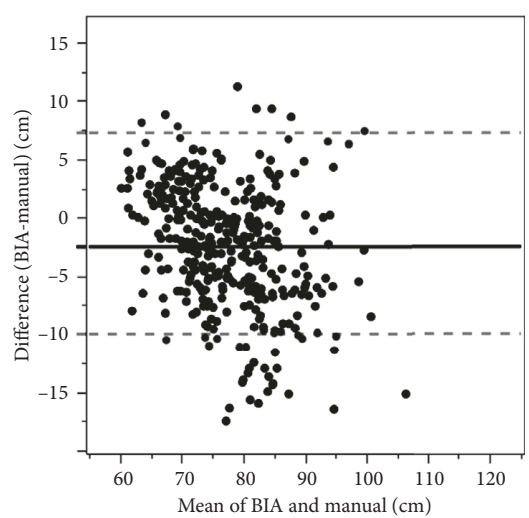

(c)

Figure 2: Bland-Altman plot of difference in WC (BIA measurement minus manual measurement) against the mean of two measurements. The middle line denotes bias (mean difference between the two measurements), and dashed lines denote 95\% limits of agreement (1.96SD of the difference). (a) Overall (bias: -2.024 , 95\% LOA: -11.87 to 7.83). (b) Male (bias: $-1.418,95 \%$ LOA: -10.03 to 7.20 ). (c) Female (bias: $-2.460,95 \%$ LOA: -13.04 to 8.12 ).

TABLE 3: Comparison between the normal group and LS risk group according to sex.

\begin{tabular}{|c|c|c|c|c|c|c|c|c|c|}
\hline \multirow[b]{2}{*}{ Variables } & \multicolumn{3}{|c|}{ Total } & \multicolumn{3}{|c|}{ Male } & \multicolumn{3}{|c|}{ Female } \\
\hline & $\begin{array}{c}\text { Normal } \\
(N=271)\end{array}$ & $\begin{array}{c}\text { LS risk } \\
(N=326)\end{array}$ & $\begin{array}{c}P \\
\text { value }\end{array}$ & $\begin{array}{c}\text { Normal } \\
(N=129)\end{array}$ & $\begin{array}{c}\text { LS risk } \\
(N=121)\end{array}$ & $\begin{array}{c}P \\
\text { value }\end{array}$ & $\begin{array}{c}\text { Normal } \\
(N=142)\end{array}$ & $\begin{array}{c}\text { LS risk } \\
(N=205)\end{array}$ & $\begin{array}{c}P \\
\text { value }\end{array}$ \\
\hline Age (years) & $63.0(9.8)$ & $66.0(10.1)$ & $<0.001$ & $64.6(9.1)$ & $68.5(9.2)$ & 0.002 & $61.5(10.3)$ & $64.6(10.3)$ & 0.007 \\
\hline Sex (male/female) & $129 / 142$ & $121 / 205$ & 0.010 & & & & & & \\
\hline Height $(\mathrm{cm})$ & $158.6(8.4)$ & $157.7(8.5)$ & 0.22 & $165.0(6.0)$ & $165.0(6.1)$ & 0.99 & $152.7(5.6)$ & $153.4(6.5)$ & 0.51 \\
\hline Weight (kg) & $58.3(11.2)$ & $59.9(11.9)$ & 0.066 & $66.1(9.5)$ & $67.7(11.9)$ & 0.22 & $51.3(7.3)$ & $55.4(9.3)$ & $<0.001$ \\
\hline BMI $\left(\mathrm{kg} / \mathrm{m}^{2}\right)$ & $23.1(3.1)$ & $24.0(3.8)$ & $<0.001$ & $24.2(2.8)$ & $24.8(3.8)$ & 0.041 & $22.0(3.0)$ & $23.6(3.7)$ & $<0.001$ \\
\hline PBF (\%) & $27.5(6.5)$ & $30.5(7.0)$ & $<0.001$ & $24.2(4.9)$ & $26.2(6.2)$ & 0.003 & $30.5(6.2)$ & $33.0(6.3)$ & $<0.001$ \\
\hline aSMI $\left(\mathrm{kg} / \mathrm{m}^{2}\right)$ & $6.8(1.1)$ & $6.7(1.0)$ & 0.46 & $7.6(0.7)$ & $7.5(0.8)$ & 0.31 & $6.0(0.6)$ & $6.2(0.8)$ & 0.006 \\
\hline WC by BIA $(\mathrm{cm})$ & $78.5(8.9)$ & $83.3(10.7)$ & 0.003 & $83.6(7.9)$ & $86.3(10.8)$ & 0.027 & $73.8(6.8)$ & $77.7(8.2)$ & $<0.001$ \\
\hline $\begin{array}{l}\text { Total cholesterol } \\
(\mathrm{mg} / \mathrm{dL})\end{array}$ & $211.3(34.7)$ & $206.3(30.9)$ & 0.11 & $206.5(35.8)$ & $200.6(31.0)$ & 0.15 & $215.6(33.1)$ & $209.7(30.4)$ & 0.22 \\
\hline $\begin{array}{l}\text { Triglycerides } \\
(\mathrm{mg} / \mathrm{dL})\end{array}$ & $101.0(57.8)$ & $112.6(70.7)$ & 0.019 & $115.1(68.7)$ & $130.1(94.5)$ & 0.25 & $88.2(42.0)$ & $102.3(49.4)$ & 0.006 \\
\hline
\end{tabular}

Evaluated using the Mann-Whitney $U$ test and Fisher's exact test. Parameter values are shown as means (standard deviations). Bold values indicate significant difference. LS, locomotive syndrome; BMI, body mass index; PBF, percent body fat; WC, waist circumference; BIA, bioelectrical impedance analysis; aSMI, appendicular skeletal muscle index.

TABLE 4: Logistic regression model for LS risk in all the participants.

\begin{tabular}{lccc}
\hline Variables & $\beta$ & Odds ratio (95\% CI) & $P$ value \\
\hline WC by BIA $(\mathrm{cm})$ & 0.102 & $1.108(1.057-1.161)$ & $<\mathbf{0 . 0 0 1}$ \\
Age (years) & 0.051 & $1.053(1.033-1.073)$ & $<\mathbf{0 . 0 0 1}$ \\
Sex (male) & -1.420 & $0.242(0.151-0.386)$ & $<\mathbf{0 . 0 0 1}$ \\
Total cholesterol (mg/dL) & -0.007 & $0.994(0.988-0.999)$ & $\mathbf{0 . 0 1 6}$ \\
BMI $\left(\mathrm{kg} / \mathrm{m}^{2}\right)$ & & & 0.051 \\
Triglycerides $(\mathrm{mg} / \mathrm{dL})$ & & & 0.20 \\
PBF $(\%)$ & & & 0.88 \\
\hline
\end{tabular}

All variables $(P<0.25)$ that showed a certain degree of difference in univariate analysis were used as covariates. The dependent variable was LS risk. Covariates were age, sex, BMI, PBF, WC by BIA, total cholesterol, and triglycerides. Bold values type indicate significant difference. LS, locomotive syndrome; $\beta$, partial regression coefficient; CI, confidence intervals; WC, waist circumference; BIA, bioelectrical impedance analysis; BMI, body mass index; PBF, percent body fat.

in a large-scale prospective health checkup population. Furthermore, the Inbody 770 BIA device has been used to measure PBF and aSMI in various studies [29, 30], so it was thought that additional evidence could be accumulated.

Because manual WC measurement introduces errors, it is necessary to evaluate the agreement with the manual method when evaluating the accuracy of WC measurement using BIA. If the agreement is high, it is possible that the measurement modalities are interchangeable. Generally, in order to validate measured values using two measurement methods, Spearman correlation and ICC are used. However, correlation analysis is a method of evaluating the relationship between two different events, and the value of the correlation coefficient cannot evaluate differences or variations between measured values. Therefore, it is difficult to evaluate interchangeability only using these assessments. It is also necessary to evaluate the agreement using BlandAltman analysis, a method of measuring by two methods and examining the differences between the measured values. In this study, the interchangeability of BIA and manual 
TABLE 5: Logistic regression model for LS risk according to sex.

\begin{tabular}{|c|c|c|c|c|c|c|c|}
\hline \multicolumn{3}{|c|}{ Male } & \multirow{2}{*}{$P$ value } & \multicolumn{3}{|c|}{ Female } & \multirow{2}{*}{$P$ value } \\
\hline Variables & $\beta$ & Odds ratio $(95 \% \mathrm{CI})$ & & Variables & $\beta$ & Odds ratio $(95 \% \mathrm{CI})$ & \\
\hline WC by BIA $(\mathrm{cm})$ & 0.095 & $1.100(1.029-1.176)$ & 0.005 & WC by BIA $(\mathrm{cm})$ & 0.134 & $1.143(1.073-1.217)$ & $<0.001$ \\
\hline Age (years) & 0.058 & $1.060(1.027-1.093)$ & $<0.001$ & Age (years) & 0.051 & $1.052(1.027-1.079)$ & $<0.001$ \\
\hline BMI $\left(\mathrm{kg} / \mathrm{m}^{2}\right)$ & & & 0.080 & Total cholesterol (mg/dL) & & & 0.065 \\
\hline Total cholesterol $(\mathrm{mg} / \mathrm{dL})$ & & & 0.24 & PBF $(\%)$ & & & 0.067 \\
\hline PBF (\%) & & & 0.48 & Triglycerides (mg/dL) & & & 0.20 \\
\hline Triglycerides (mg/dL) & & & 0.52 & $\operatorname{aSMI}\left(\mathrm{kg} / \mathrm{m}^{2}\right)$ & & & 0.25 \\
\hline & & & & BMI $\left(\mathrm{kg} / \mathrm{m}^{2}\right)$ & & & 0.44 \\
\hline
\end{tabular}

All variables $(P<0.25)$ that showed a certain degree of difference in univariate analysis were used as covariates. The dependent variable was LS risk. Covariates in males were age, BMI, PBF, WC by BIA, total cholesterol, and triglycerides. Covariates in females were age, BMI, PBF, aSMI, WC by BIA, total cholesterol, and triglycerides. Bold values indicate significant difference. $\beta$, partial regression coefficient; LS, locomotive syndrome; CI, confidence intervals; WC, waist circumference; BIA, bioelectrical impedance analysis; BMI, body mass index; PBF, percent body fat; aSMI, appendicular skeletal muscle index.

measurements was also evaluated based on past reports [23]. Then, for males and females, there was a high correlation between WC measured using BIA and manual methods, demonstrating interchangeability. This result is very important, and there is the possibility for future research can focus on the use of BIA not only for WC but also other trunk circumferences.

Moreover, because we confirmed that WC measurement by BIA is interchangeable with manual measurement, our previous report [6] was further developed and examined. In that report, we did not evaluate males and LS risk was not considered. The present study differs from our previous study in the following ways: we measure WC using BIA as well as the manual method; we increase the number of subjects, including males; and we include total cholesterol and triglyceride, as well as the evaluation of LS risk which is an indicator of mobility. With respect to the presence or absence of LS risk, the aim was to include an earlier stage; in the past, relevant factors have been investigated in various approaches in the same way $[4,5]$. We found in univariate analysis that WC measurement by BIA was significantly high in the LS risk group overall, in males and in females. Furthermore, even if statistical adjustments were made for other factors in logistic regression analysis, the increase in WC measured by BIA was found to be a significant LS risk factor. This study has allowed us to further develop the previous report and to accumulate further evidence. It is important to continue focusing on the increase of $\mathrm{WC}$ and to improve early detection of LS risk.

This study has several limitations. First, it was a singlecenter study and, therefore, may be subject to selection bias. Multicenter studies are needed to validate our findings. Second, measurement differences may occur if BIA devices from different manufacturers are used. Therefore, standardization of technology and cross calibration of electrical resistance should be addressed in the future.

\section{Conclusions}

We found that WC measured by BIA (Inbody 770) is interchangeable with manual measurements. We also showed that even with statistical corrections for various relevant factors, the increases in WC measured by BIA were significantly associated with LS risk not only in females but also in males. The progress of BIA technology is remarkable; depending on the device, it is possible to measure trunk circumferences other than WC, and a large amount of data can be obtained in a short time using one measurement. Therefore, according to the results of this study, it may be possible to investigate on a large scale the relationship between trunk circumferences including WC and various diseases and conditions.

\section{Data Availability}

The data used to support the findings of this study are included within the article.

\section{Ethical Approval}

The study protocol was approved by the Institutional Review Board of Nagoya University Graduate School of Medicine. Moreover, the study protocol was approved by the Committee on Ethics in Human Research of our university, and the study procedures were carried out in accordance with the principles of the Declaration of Helsinki.

\section{Consent}

All participants provided written informed consent.

\section{Conflicts of Interest}

Satoshi Tanaka, Kei Ando, Kazuyoshi Kobayashi, Taisuke Seki, Shinya Ishizuka, Masaaki Machino, Masayoshi Morozumi, Shunsuke Kanbara, Sadayuki Ito, Taro Inoue, Naoki Ishiguro, Yukiharu Hasegawa, and Shiro Imagama declare that they have no conflicts of interest.

\section{Acknowledgments}

We are grateful to the staff of the Comprehensive Health Care Program held in Yakumo, Hokkaido, and to Ms. Aya Hemmi and Ms. Hiroko Ino of Nagoya University for their assistance throughout this study. 


\section{References}

[1] K. Nakamura, "The concept and treatment of locomotive syndrome: its acceptance and spread in Japan," Journal of Orthopaedic Science, vol. 16, no. 5, pp. 489-491, 2011.

[2] S. Imagama, Y. Hasegawa, K. Ando et al., "Staged decrease of physical ability on the locomotive syndrome risk test is related to neuropathic pain, nociceptive pain, shoulder complaints, and quality of life in middle-aged and elderly people-the utility of the locomotive syndrome risk test," Modern Rheumatology, vol. 27, no. 6, pp. 1051-1056, 2017.

[3] N. Yoshimura, S. Muraki, H. Oka et al., "Association between new indices in the locomotive syndrome risk test and decline in mobility: third survey of the ROAD study," Journal of Orthopaedic Science, vol. 20, no. 5, pp. 896-905, 2015.

[4] S. Tanaka, K. Ando, K. Kobayashi et al., "Increasing postural sway in balance test is related to locomotive syndrome risk: a cross-sectional study," Journal of Orthopaedic Science, vol. 24, no. 5, pp. 912-917, 2019.

[5] S. Tanaka, K. Ando, K. Kobayashi et al., "Serum cystatin C level is associated with locomotive syndrome risk and can be an early predictor in community-living people: the Yakumo study," Modern Rheumatology, vol. 28, no. 6, pp. 1035-1040, 2018.

[6] A. Muramoto, S. Imagama, Z. Ito et al., "Waist circumference is associated with locomotive syndrome in elderly females," Journal of Orthopaedic Science, vol. 19, no. 4, pp. 612-619, 2014.

[7] S. Tanaka, K. Ando, K. Kobayashi et al., "The decrease in phase angle measured by bioelectrical impedance analysis reflects the increased locomotive syndrome risk in communitydwelling people: the Yakumo study," Modern Rheumatology, vol. 29, no. 3, pp. 496-502, 2019.

[8] S. Tanaka, K. Ando, K. Kobayashi et al., "The decreasing phase angles of the entire body and trunk during bioelectrical impedance analysis are related to locomotive syndrome," Journal of Orthopaedic Science, vol. 24, no. 4, pp. 720-724, 2019.

[9] S. Tanaka, K. Ando, K. Kobayashi et al., "Relationship between locomotive syndrome and body composition among community-dwelling middle-age and elderly individuals in Japan: the Yakumo study," Modern Rheumatology, vol. 29, no. 3, pp. 491-495, 2019.

[10] J. M. Bland and D. G. Altman, "Statistical methods for assessing agreement between two methods of clinical measurement," The Lancet, vol. 327, no. 8476, pp. 307-310, 1986.

[11] S. Imagama, Y. Hasegawa, Y. Matsuyama et al., "Influence of sagittal balance and physical ability associated with exercise on quality of life in middle-aged and elderly people," Archives of Osteoporosis, vol. 6, no. 1-2, pp. 13-20, 2011.

[12] S. Imagama, Y. Matsuyama, Y. Hasegawa et al., "Back muscle strength and spinal mobility are predictors of quality of life in middle-aged and elderly males," European Spine Journal, vol. 20, no. 6, pp. 954-961, 2011.

[13] S. Tanaka, K. Ando, K. Kobayashi et al., "A low phase angle measured with bioelectrical impedance analysis is associated with osteoporosis and is a risk factor for osteoporosis in community-dwelling people: the Yakumo study," Archives of Osteoporosis, vol. 13, no. 1, p. 39, 2018.

[14] S. Tanaka, K. Ando, K. Kobayashi et al., "Utility of the serum cystatin C level for diagnosis of osteoporosis among middleaged and elderly people," BioMed Research International, vol. 2019, Article ID 5046852, 6 pages, 2019.

[15] U. G. Kyle, I. Bosaeus, A. D. De Lorenzo et al., "Bioelectrical impedance analysis-part II: utilization in clinical practice," Clinical Nutrition, vol. 23, no. 6, pp. 1430-1453, 2004.
[16] A. Seichi, Y. Hoshino, T. Doi, M. Akai, Y. Tobimatsu, and T. Iwaya, "Development of a screening tool for risk of locomotive syndrome in the elderly: the 25 -question geriatric locomotive function scale," Journal of Orthopaedic Science, vol. 17, no. 2, pp. 163-172, 2012.

[17] S. Imagama, Y. Hasegawa, N. Wakao, K. Hirano, A. Muramoto, and N. Ishiguro, "Impact of spinal alignment and back muscle strength on shoulder range of motion in middle-aged and elderly people in a prospective cohort study," European Spine Journal, vol. 23, no. 7, pp. 1414-1419, 2014.

[18] S. Tanaka, K. Ando, K. Kobayashi et al., "Reduction in body cell mass as a predictor of osteoporosis: a cross-sectional study," Modern Rheumatology, pp. 1-6, 2019.

[19] S. Tanaka, K. Ando, K. Kobayashi et al., "Low bioelectrical impedance phase Angle is a significant risk factor for frailty," BioMed Research International, vol. 2019, Article ID 6283153, 7 pages, 2019.

[20] S. Tanaka, K. Ando, K. Kobayashi et al., "Declining neck circumference is an anthropometric marker related to frailty in middle-aged and elderly women," Modern Rheumatology, pp. 1-7, 2019.

[21] D.-H. Lee, K. Park, S. Ahn et al., "Comparison of abdominal visceral adipose tissue area measured by computed tomography with that estimated by bioelectrical impedance analysis method in Korean subjects," Nutrients, vol. 7, no. 12, pp. 10513-10524, 2015.

[22] K. S. Park, D.-H. Lee, J. Lee et al., "Comparison between two methods of bioelectrical impedance analyses for accuracy in measuring abdominal visceral fat area," Journal of Diabetes and its Complications, vol. 30, no. 2, pp. 343-349, 2016.

[23] T. Hida, S. Imagama, K. Ando et al., "Sarcopenia and physical function are associated with inflammation and arteriosclerosis in community-dwelling people: the Yakumo study," Modern Rheumatology, vol. 28, no. 2, pp. 345-350, 2018.

[24] J. R. Landis and G. G. Koch, "The measurement of observer agreement for categorical data," Biometrics, vol. 33, no. 1, pp. 159-174, 1977.

[25] L. A. H. Critchley and J. A. J. H. Critchley, "A meta-analysis of studies using bias and precision statistics to compare cardiac output measurement techniques," Journal of Clinical Monitoring and Computing, vol. 15, no. 2, pp. 85-91, 1999.

[26] T. Hayashi, E. J. Boyko, D. L. Leonetti et al., "Visceral adiposity is an independent predictor of incident hypertension in Japanese Americans," Annals of Internal Medicine, vol. 140, no. 12, pp. 992-1000, 2004.

[27] C. K. Kramer, D. von Mühlen, J. L. Gross, and E. BarrettConnor, "A prospective study of abdominal obesity and coronary artery calcium progression in older adults," The Journal of Clinical Endocrinology \& Metabolism, vol. 94, no. 12, pp. 5039-5044, 2009.

[28] M. Ochi, Y. Tabara, T. Kido et al., "Quadriceps sarcopenia and visceral obesity are risk factors for postural instability in the middle-aged to elderly population," Geriatrics \& Gerontology International, vol. 10, no. 3, pp. 233-243, 2010.

[29] C. N. McLester, B. S. Nickerson, B. M. Kliszczewicz, and J. R. McLester, "Reliability and agreement of various InBody body composition analyzers as compared to dual-energy X-ray absorptiometry in healthy men and women," Journal of Clinical Densitometry, vol. 38, pp. 10-27, 2018.

[30] S. Lee, S. Ahn, Y. Kim et al., "Comparison between dualenergy X-ray absorptiometry and bioelectrical impedance analyses for accuracy in measuring whole body muscle mass and appendicular skeletal muscle mass," Nutrients, vol. 10, no. 6, p. 738, 2018. 


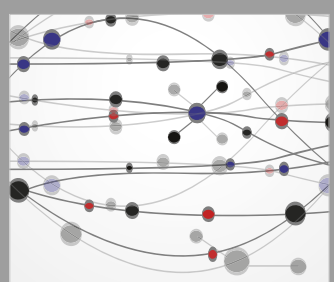

The Scientific World Journal
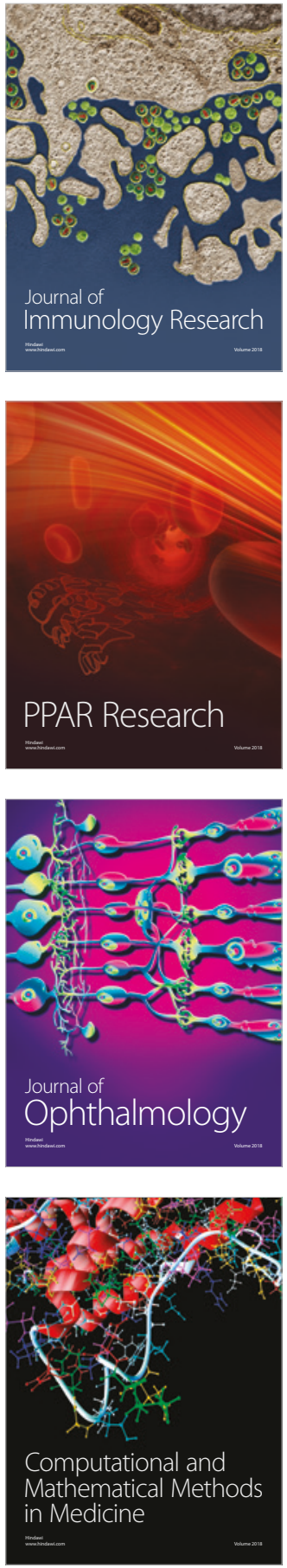

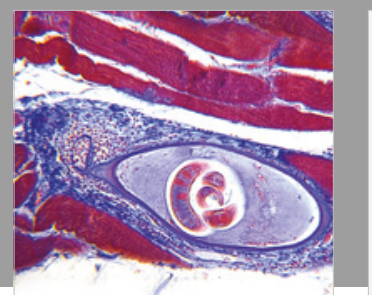

Gastroenterology Research and Practice

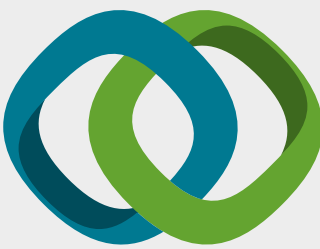

\section{Hindawi}

Submit your manuscripts at

www.hindawi.com
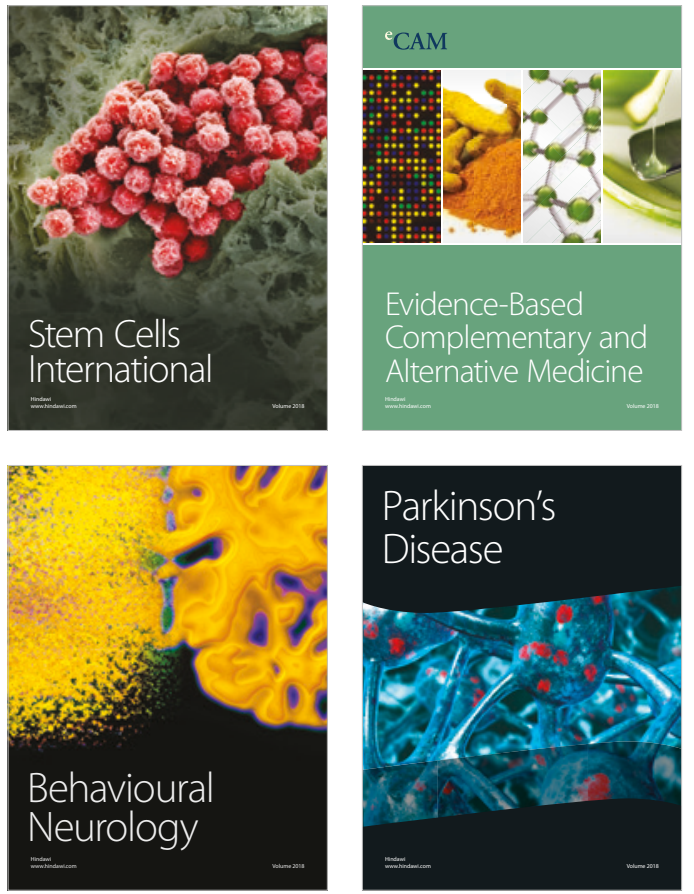

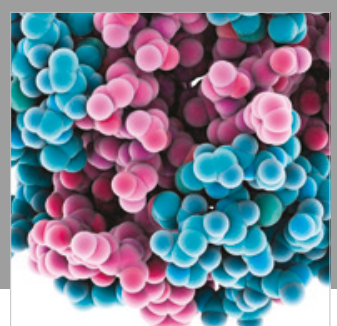

ournal of

Diabetes Research

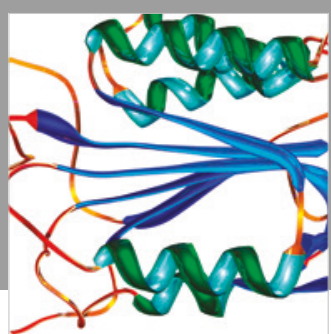

Disease Markers
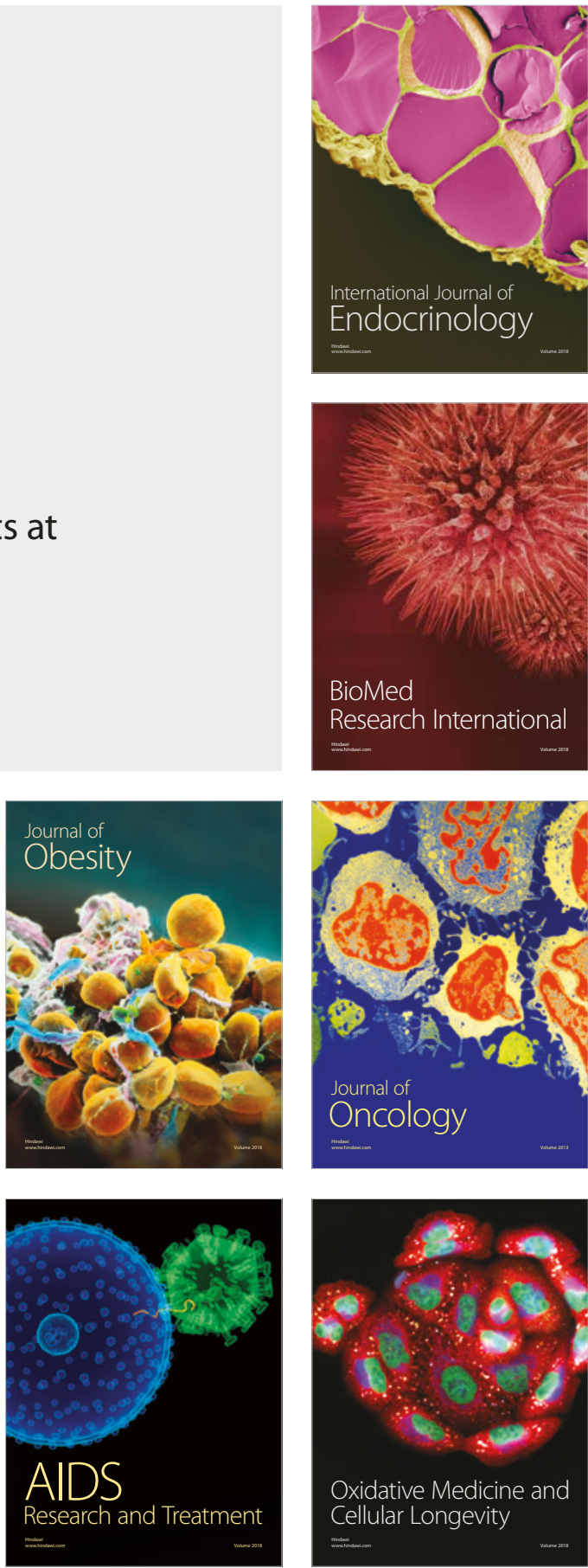